Matthias Eller (Washington, DC)

\title{
SYMMETRIC HYPERBOLIC SYSTEMS WITH BOUNDARY CONDITIONS THAT DO NOT SATISFY THE KREISS-SAKAMOTO CONDITION
}

Abstract. Symmetric hyperbolic systems with a class of non-homogeneous boundary conditions that do not satisfy the Kreiss-Sakamoto condition (or uniform Lopatinskil condition) are discussed. The boundary conditions are of conservative type. An energy estimate which provides interior and boundary regularity for weak solutions to the system is proved. The results are valid for operators with rough coefficients. As an example the anisotropic Maxwell system is considered.

1. Introduction and main result. Consider a system of partial differential equations of the form

$$
\begin{array}{r}
P u:=E(t, x) \partial_{t} u(t, x)+A^{j}(t, x) \partial_{j} u(t, x)+B(t, x) u(t, x)=f(t, x), \\
(t, x) \in Q:=(0, T) \times \Omega,
\end{array}
$$

where $\Omega$ is a connected subset of $\mathbb{R}^{n}$ with a $C^{2}$-boundary $\Gamma=\partial \Omega, E(t, x)$ and $A(t, x)$ are Hermitian $N \times N$ matrix functions, the matrix $B(t, x)$ is $N \times N$, and $u$ and $f$ are vector-valued functions of $n+1$ variables with $N$ components. Here and henceforth we use the summation convention: terms with repeated indices are added from 1 to $n$. Furthermore, the matrix $E$ is assumed to be uniformly positive definite on $Q$ and we write $\partial_{t}=\partial / \partial t$ and $\partial_{j}=\partial / \partial x_{j}$. The variable $t \in \mathbb{R}$ is the time variable and $x \in \mathbb{R}^{n}$ is the space variable, and we fix a final time $T>0$.

This kind of system is called symmetric hyperbolic [F54]. We add a boundary condition

2000 Mathematics Subject Classification: Primary 35L50.

Key words and phrases: symmetric hyperbolic systems, boundary value problems, uniform Lopatinskiı̌ condition.

Research supported by the National Science Foundation through grant DMS-0606118. 


$$
M(t, x) u(t, x)=g(t, x), \quad(t, x) \in \Sigma:=(0, T) \times \Gamma,
$$

where $M$ is a $N \times N$ matrix, $g=0$ on $\operatorname{Ker} M$, and an initial condition

$$
u(0, x)=u_{0}(x),
$$

and consider the initial-boundary value problem (1.1)-(1.3).

We start with a brief discussion of dissipative boundary conditions [LP60]. Let $\nu=\left(\nu_{1}, \ldots, \nu_{n}\right)^{T}$ be the exterior unit normal of $\Omega$ on $\Gamma$. Applying a unitary transformation to the dependent variable $u$ we can assume that $A^{j} \nu_{j}$ is in (block-)diagonal form:

$$
A^{j} \nu_{j}=\left(\begin{array}{rrr}
0 & 0 & 0 \\
0 & \Lambda_{1}^{2} & 0 \\
0 & 0 & -\Lambda_{2}^{2}
\end{array}\right)
$$

at every point $(t, x) \in \Sigma$, where $\Lambda_{1}$ and $\Lambda_{2}$ are Hermitian, positive definite matrices. In what follows we will assume that $\Lambda_{1}=\Lambda_{1}(t, x)$ and $\Lambda_{2}=$ $\Lambda_{2}(t, x)$ are both $k \times k$ matrices with $L_{\infty}$ entries and $2 k \leq N$. Corresponding to this decomposition of $A^{j} \nu_{j}$ we decompose $u=\left(z, u_{1}, u_{2}\right)$.

Let $\mathcal{O}(t, x) \in L_{\infty}(\Sigma)$ be a $k \times k$ matrix such that $|\mathcal{O} h| \leq c|h|$ for all $h \in \mathbb{C}^{k}$ almost everywhere on $\Sigma$. Here $c<1$ is a positive constant and $|\cdot|$ denotes the Euclidean norm in $\mathbb{C}^{k}$. The boundary condition (1.2) is dissipative if

$$
M=\left(\begin{array}{rrr}
0 & 0 & 0 \\
0 & 0 & 0 \\
0 & \mathcal{O} \Lambda_{1} & \Lambda_{2}
\end{array}\right), \quad M u=\Lambda_{2} u_{2}+\mathcal{O} \Lambda_{1} u_{1} .
$$

We will assume that the boundary matrix $M$ is $k \times 2 k$. From (1.4) one can derive the inequality

$$
\frac{1-c^{2}}{2}\left|\Lambda_{1} u_{1}\right|^{2} \leq u^{H}\left[A^{j} \nu_{j}\right] u+\frac{1+c^{2}}{1-c^{2}}|M u|^{2}
$$

almost everywhere on $\Sigma$, where $u^{H}$ denotes the Hermitian transpose of the (column) vector $u$. Using integration by parts (see formula (2.1) below) one shows that a sufficiently regular solution to the boundary value problem (1.1)-(1.3) satisfies the estimate

$$
\begin{aligned}
\left\|e^{-\gamma T} u(T)\right\|_{L_{2}(\Omega)}^{2}+ & \gamma\left\|e^{-\gamma t} u\right\|_{L_{2}(Q)}^{2}+\left|e^{-\gamma t} u\right|_{L_{2}(\Sigma)}^{2} \\
& \widetilde{\leq}\left\|u_{0}\right\|_{L_{2}(\Omega)}^{2}+\frac{1}{\gamma}\left\|e^{-\gamma t} f\right\|_{L_{2}(Q)}^{2}+\left|e^{-\gamma t} g\right|_{L_{2}(\Sigma)}^{2}
\end{aligned}
$$

for $\gamma \geq \gamma_{0}$ provided that $\operatorname{det} A^{j} \nu_{j} \geq c>0$ almost everywhere on $\Sigma$. Here $a \widetilde{\leq} b$ means $a \leq C b$ for some constant $C$. 
In 1970 Kreiss showed that for strictly hyperbolic systems the estimate (1.5) is valid as long as the boundary condition satisfies the Kreiss-Sakamoto condition [K70]. At the same time, Sakamoto proved an analogous result for scalar hyperbolic equations [S70].

In this note we discuss a special class of boundary conditions which does not satisfy the Kreiss-Sakamoto condition (or uniform Lopatinskil condition). We say the boundary condition (1.2) is conservative if

$$
M u=\Lambda_{2} u_{2}+U \Lambda_{1} u_{1}
$$

where $U=U(t, x)$ is a unitary $k \times k$ matrix with $L_{\infty}$ entries. In the case of the homogeneous boundary condition $M u=0$ one can derive the estimate (1.5) without boundary integrals since

$\left\langle A^{j} \nu_{j} u, u\right\rangle_{\Sigma}=\left\langle U \Lambda_{1} u_{1}, U \Lambda_{1} u_{1}\right\rangle_{\Sigma}-\left\langle\Lambda_{2} u_{2}, \Lambda_{2} u_{2}\right\rangle_{\Sigma}=\Re\left\langle M u, U \Lambda_{1} u_{1}-\Lambda_{2} u_{2}\right\rangle_{\Sigma}$ where $\langle\cdot, \cdot\rangle_{\Sigma}$ denotes the scalar product in $L_{2}(\Sigma)$.

Conservative boundary conditions are of interest in applied problems. The Neumann boundary condition is a conservative boundary condition for the scalar wave equation, and the perfect conductor boundary condition $\nu \times e=0$ is conservative for Maxwell's equations. In both cases it is known that the Kreiss-Sakamoto condition does not hold [M73, p. 439], [CP82, Chapter 7, Example 3.11], [MO75, Section 2], which is to say that the inhomogeneous boundary value problem is not well-posed in $L_{2}$ in the sense of estimate (1.5). However, a weaker form of the Kreiss-Sakamoto condition, the Lopatinskil condition is still satisfied and provides solutions without estimates to both problems in the case that $\Omega$ is the half-space $\mathbb{R}_{+}^{n}=\left\{x_{n} \geq 0\right\}$ and the operator has constant coefficients.

The only problem of this type which has received a lot of attention is the Neumann problem for scalar second order hyperbolic equations. Miyatake proved estimates for this problem and observed that higher regularity of the boundary data is required to obtain a finite energy solution [M73]. Later Lasiecka and Triggiani gave a very detailed treatment of this Neumann problem, in particular with $L_{2}(\Sigma)$ boundary conditions [LT90], [LT91], [LT94], establishing sharp interior and boundary regularity in many cases. More recently, Tataru refined their boundary regularity results [T98].

Our research was motivated by the search for a regularity result for Maxwell's system with a non-homogeneous perfect conductor boundary condition. In a private communication with the author, Belishev has pointed out that a sharp regularity result for this boundary value problem is not available.

Our main results are the following two theorems. Here $H^{s}$ stands for the $L_{2}$-based Sobolev space of order $s$. 
THEOREM 1.1. Assume that $u \in H^{1}(Q)$ is a weak solution of the symmetric hyperbolic system (1.1) where the entries of $E$ and $A^{1}, \ldots, A^{n}$ are of class $C^{1}$ and the entries of $B$ are in $L_{\infty}(Q)$. Assume that the boundary condition (1.2) is conservative in the sense that (1.6) is valid and that the boundary is uniformly non-characteristic, i.e. $\left|\operatorname{det}\left(A^{j} \nu_{j}\right)\right| \geq c>0$ for all $(t, x) \in \Sigma$. Then there exists a constant $\gamma_{0}$ such that

$$
\begin{aligned}
& \left\|e^{-\gamma T} u(T)\right\|_{L_{2}(\Omega)}^{2}+\gamma\left\|e^{-\gamma t} u\right\|_{L_{2}(Q)}^{2}+\left|e^{-\gamma t} u\right|_{L_{2}\left(0, T ; H^{-1 / 2}(\Gamma)\right)}^{2} \\
& \quad \widetilde{\leq}\|u(0)\|_{L_{2}(\Omega)}^{2}+\frac{1}{\gamma}\left\|e^{-\gamma t} P u\right\|_{L_{2}(Q)}^{2}+\left|e^{-\gamma t} M u\right|_{L_{2}\left(0, T ; H^{1 / 2}(\Gamma)\right)}^{2}
\end{aligned}
$$

for $\gamma \geq \gamma_{0}$.

The second theorem considers the case of a characteristic boundary.

Theorem 1.2. Assume that $u \in H^{1}(Q)$ is a weak solution of the symmetric hyperbolic system (1.1) where the entries of $E$ and $A^{1}, \ldots, A^{n}$ are of class $C^{1}$ and the entries of $B$ are in $L_{\infty}(Q)$. Assume that the boundary condition (1.2) is conservative and that the boundary is uniformly characteristic, i.e. $\operatorname{rank}\left(A^{j} \nu_{j}\right)=N^{\prime}<N$ for all $(t, x) \in \Sigma$. Then there exists a constant $\gamma_{0}$ such that

$$
\begin{aligned}
\left\|e^{-\gamma T} u(T)\right\|_{L_{2}(\Omega)}^{2}+\gamma\left\|e^{-\gamma t} u\right\|_{L_{2}(Q)}^{2}+\left|e^{-\gamma t} \Pi u\right|_{L_{2}\left(0, T ; H^{-1 / 2}(\Gamma)\right)}^{2} \\
\quad \widetilde{\leq}\|u(0)\|_{L_{2}(\Omega)}^{2}+\frac{1}{\gamma}\left\|e^{-\gamma t} P u\right\|_{L_{2}(Q)}^{2}+\left|e^{-\gamma t} M u\right|_{L_{2}\left(0, T ; H^{1 / 2}(\Gamma)\right)}^{2}
\end{aligned}
$$

for $\gamma \geq \gamma_{0}$. Here $\Pi$ denotes the orthogonal projection from $\mathbb{C}^{n}$ onto $\left[\operatorname{Ker} A^{j} \nu_{j}\right]^{\perp}$.

The estimates (1.7) and (1.8) can be used to establish existence, uniqueness, and regularity for the initial value problem (1.1)-(1.3). This can be proved by first proving uniqueness for the (backward) adjoint problem with homogeneous boundary data and then solving the original problem by means of duality.

Theorem 1.3. Given $f \in L_{2}(Q), g \in L_{2}\left(0, T ; H^{1 / 2}(\Gamma)\right)$ and $u_{0} \in L_{2}(\Omega)$ there exists a unique solution $u \in C\left([0, T], L_{2}(\Omega)\right)$ to the initial-boundary value problem (1.1)-(1.3), and the mapping

$$
\left(f, g, u_{0}\right) \mapsto\left(u,\left.\Pi u\right|_{\Sigma}\right)
$$

is continuous from $L_{2}(Q) \times L_{2}\left(0, T ; H^{1 / 2}(\Gamma)\right) \times L_{2}(\Omega)$ into $C\left([0, T], L_{2}(\Omega)\right) \times$ $L_{2}\left(0, T ; H^{-1 / 2}(\Gamma)\right)$.

ExAmple 1. The dynamic Maxwell equations for the electric field intensity $e$ and the magnetic field intensity $h$ are

$$
\begin{aligned}
\varepsilon \partial_{t} e-\nabla \times h+\sigma e & =f_{1}, \\
\mu \partial_{t} h+\nabla \times e & =f_{2},
\end{aligned}
$$


where the electric permittivity $\varepsilon$ and the magnetic permeability $\mu$ are positive definite, Hermitian $3 \times 3$ matrices and the conductivity $\sigma$ is a $3 \times 3$ matrix. If $\varepsilon, \mu$ and $\sigma$ are multiples of the identity matrix, then we say that the system is isotropic. Otherwise we refer to it as anisotropic. We add the initial condition

$$
e(0)=e_{0}, \quad h(0)=h_{0}
$$

and the boundary condition

$$
\nu \times e=g \quad \text { on } \Sigma
$$

where $\nu \cdot g=0$ on $\Sigma$. Note that this boundary is characteristic since

$$
A^{j} \nu_{j}(e, h)=(-\nu \times h, \nu \times e)
$$

and $\operatorname{rank}\left(A^{j} \nu_{j}\right)=4$. The projection $\Pi$ defined in Theorem 1.2 is the projection of $(e, h)$ onto its tangential components. The boundary condition (1.11) is conservative since

$$
\left\langle A^{j} \nu_{j}(e, h),(e, h)\right\rangle_{\Sigma}=2 \Re\left\langle\nu \times e, h_{\tau}\right\rangle_{\Sigma}=2 \Re\left\langle g, h_{\tau}\right\rangle_{\Sigma} .
$$

Here $h_{\tau}=(\nu \times h) \times \nu$ denotes the tangential component of $h$ on $\Sigma$. From Theorems 1.2 and 1.3 we obtain the following statement for the initialboundary value problem (1.9)-(1.11).

COROLlary 1.4. If $\varepsilon$ and $\mu$ have $C^{1}$-entries, $\sigma$ has entries in $L_{\infty}, f \in$ $L_{2}(Q), e_{0}, h_{0} \in L_{2}(\Omega)$, and $g \in L_{2}\left(0, T ; H^{1 / 2}(\Gamma)\right)$, then there exists a unique solution $(e, h) \in C\left([0, T], L_{2}(\Omega)\right)$ to (1.9)-(1.11) and the following estimate holds:

$$
\begin{aligned}
& \|(e, h)\|_{C\left([0, T], L_{2}(\Omega)\right)}^{2}+\left|h_{\tau}\right|_{L_{2}\left(0, T ; H^{-1 / 2}(\Gamma)\right)}^{2} \\
& \widetilde{\leq}\left\|\left(e_{0}, h_{0}\right)\right\|_{L_{2}(\Omega)}^{2}+\|f\|_{L_{2}(Q)}^{2}+|g|_{L_{2}\left(0, T ; H^{1 / 2}(\Gamma)\right)}^{2} .
\end{aligned}
$$

This estimate improves results given earlier for the isotropic Maxwell system by Majda and Osher [MO75, Proposition 2.3], and more recently by Glasman [G01, Theorem 1]. For an existence and uniqueness result in a larger space than $L_{2}$ we refer to [EM02, Proposition 2.2].

Note that (1.12) does not establish a regularity result for the normal components of $e$ and $h$ on $\Sigma$. If we have the additional information $\nabla \cdot(\varepsilon e)$, $\nabla \cdot(\mu h) \in C\left([0, T], L_{2}(\Omega)\right)$, then $\nu \cdot(\varepsilon e), \nu \cdot(\mu h) \in C\left([0, T], H^{-1 / 2}(\Gamma)\right)$ by trace theory [C96, Chapter 2].

EXAMPLE 2 (The wave equation with variable coefficients and Neumann boundary conditions). Consider the equation

$$
\partial_{t}^{2} \phi-\nabla \cdot(\alpha(t, x) \nabla \phi)=\partial_{t}^{2} \phi-\partial_{j}\left(\alpha^{j k}(t, x) \partial_{k} \phi\right)=f
$$

where $\alpha$ is a positive definite, Hermitian $n \times n$ matrix. The initial condition 
is

$$
\phi(0)=\phi_{0}, \quad \partial_{t} \phi(0)=\psi_{0}
$$

and the boundary condition is

$$
\nu \cdot(\alpha \nabla \phi)=g \quad \text { on } \Sigma .
$$

The equation (1.13) is transformed into a symmetric hyperbolic system by setting

$$
u=\left(\partial_{t} \phi, \alpha \nabla \phi\right)^{T}, \quad A^{j} \partial_{j}=\left(\begin{array}{cc}
0 & -\nabla^{T} \\
-\nabla & 0
\end{array}\right), \quad E=\left(\begin{array}{cc}
1 & 0 \\
0 & \alpha^{-1}
\end{array}\right)
$$

A problem with this transformation is that it makes the boundary $\Sigma$ characteristic. One computes $\operatorname{rank}\left(A^{j} \nu_{j}\right)=2$ and

$$
\left\langle A^{j} \nu_{j} u, u\right\rangle_{\Sigma}=-2 \Re\left\langle\nu \cdot \alpha \nabla \phi, \partial_{t} \phi\right\rangle_{\Sigma} .
$$

Theorems 1.2 and 1.3 applied to this system provide the following.

COROLlary 1.5. If $\alpha$ is a symmetric, positive definite matrix with $C^{1}$ entries, $f \in L_{2}(Q),\left(\phi_{0}, \psi_{0}\right) \in H^{1}(\Omega) \times L_{2}(\Omega)$, and $g \in L_{2}\left(0, T ; H^{1 / 2}(\Gamma)\right)$, then there exists a unique solution $u \in C\left([0, T], H^{1}(\Omega)\right) \cap C^{1}\left([0, T], L_{2}(\Omega)\right)$, and the following estimate holds:

$$
\begin{aligned}
\left\|\left(\phi, \phi_{t}\right)\right\|_{C\left([0, T], H^{1}(\Omega) \times L_{2}(\Omega)\right)}^{2}+\left|\partial_{t} \phi\right|_{L_{2}\left(0, T ; H^{-1 / 2}(\Gamma)\right)}^{2} & \\
& \leq\left\|\left(\phi_{0}, \psi_{0}\right)\right\|_{H^{1}(\Omega) \times L_{2}(\Omega)}^{2}+\|f\|_{L_{2}(Q)}^{2}+|g|_{L_{2}\left(0, T ; H^{1 / 2}(\Gamma)\right)}^{2}
\end{aligned}
$$

This result is similar to the one given by Miyatake [M73, Theorem 1]. Note that we only obtain an estimate of the boundary trace of the velocity $\partial_{t} \phi$ and no estimate for the spatial tangential derivatives. However, the regularity of the trace of $\phi$ on $\Sigma$ can be established using the trace theorem for the Sobolev space $H^{1}$. The estimate (1.16) is valid for operators with $C^{1}$ coefficients, whereas Miyatake requires $C^{\infty}$ coefficients.

Before we present the proofs, we make a few comments. Most regularity results for hyperbolic equations and systems have been proved for the case that $\Omega$ is the half-space. After establishing the estimates in the half-space one can use local charts and a partition of unity and transfer the estimates to an arbitrary set $\Omega$ with a sufficiently smooth boundary. Kreiss proved the estimate (1.5) for strictly hyperbolic systems by first transforming the operator micro-locally into a special block-diagonal structure and then constructing a symmetrizer [K70], [CP82]. Métivier observed that the strict hyperbolicity assumption can be relaxed and he proved the Kreiss block structure condition for symmetrizable hyperbolic systems of constant multiplicity [M00]. More recently, Métivier and Zumbrun investigated symmetric hyperbolic systems with variable multiplicities [MZ05]. They noted that the Kreiss block structure condition is not satisfied for the anisotropic Maxwell 
system. Moreover, it is not clear how to adjust the construction of the symmetrizer when the boundary condition does not satisfy the Kreiss-Sakamoto condition.

Since our research was motivated by Maxwell's system we had to follow a different approach. Instead of moving the problem into the half-space and block-diagonalizing the symbol of the operator micro-locally we work with the symmetric system directly. This has the advantage that we do not need to construct a symmetrizer. Instead we construct a special pseudodifferential multiplier which leaves important symmetries of the system in place.

2. Proofs. For brevity we will indicate the scalar product and norm in $L_{2}(Q)$ and $L_{2}(\Omega)$ by the subscript $Q$ and $\Omega$, respectively. We will also restrict ourselves to real quantities in an effort to keep the proofs transparent.

2.1. Proof of Theorem 1.1

FIRST STEP: The multiplier $e^{-\gamma t} u$. We multiply the system (1.1) by $e^{-\gamma t}$ and take then the $L_{2}$ scalar product with $v=e^{-\gamma t} u$ over $Q$ :

$$
\left(E \partial_{t} v, v\right)_{Q}+\gamma(E v, v)_{Q}+\left(A^{j} \partial_{j} v, v\right)_{Q}+(B v, v)_{Q}=\left(e^{-\gamma t} f, v\right)_{Q} .
$$

By the symmetry of $E$ and $A^{j}$ for $j=1, \ldots, n$ and integration by parts we have

$$
(E v(T), v(T))_{\Omega}+2 \gamma(E v, v)_{Q}
$$

$$
=(E v(0), v(0))_{\Omega}+2\left(e^{-\gamma t} f, v\right)_{Q}-\left\langle A^{j} \nu_{j} v, v\right\rangle_{\Sigma}-\left(\left(2 B-\partial_{t} E-\partial_{j} A^{j}\right) v, v\right)_{Q} \text {. }
$$

Using now the fact that $E$ is positive definite and the Cauchy-Schwarz inequality, and noting that the boundary condition (1.2) satisfies relation (1.6) we arrive at

$$
\begin{aligned}
& \|v(T)\|_{\Omega}^{2}+\gamma\|v\|_{Q}^{2} \\
& \quad \widetilde{\leq}\|v(0)\|_{\Omega}^{2}+\frac{1}{\gamma}\left\|e^{-\gamma t} f\right\|_{Q}^{2}-\left\langle M v, U \Lambda_{1} v_{1}-\Lambda_{2} v_{2}\right\rangle_{\Sigma} \quad \text { for } \gamma \geq \gamma_{0},
\end{aligned}
$$

where $v_{j}=e^{-\gamma t} u_{j}$ for $j=1,2$. Since the boundary integral can be estimated by some constant times

$$
|M v|_{L_{2}\left(0, T ; H^{1 / 2}(\Gamma)\right)}^{2}+|v|_{L_{2}\left(0, T ; H^{-1 / 2}(\Gamma)\right)}^{2},
$$

we need to find a suitable estimate for the norm $|v|_{L_{2}\left(0, T ; H^{-1 / 2}(\Gamma)\right)}^{2}$. For this we construct a special multiplier.

SECOND STEP: The collar operator $q$. Since $\Gamma \in C^{2}$, there exists a tubular neighborhood $V$ of $\Gamma$ in $\mathbb{R}^{3}$. This means that for $x \in \Gamma$ and $s \in(-a, a)$ where $a$ is some real number, the map $F(x, s)=x-s \nu(x)$ is a $C^{1}$ diffeomorphism between $\Gamma \times(-a, a)$ and $V$. The set $\Omega \cap U$ is the collar of $\partial \Omega$ 
in $\Omega$. The collar is also the union of a family of closed surfaces $\Gamma_{s}$ where

$$
\Gamma_{s}=\{y \in \Omega: y=F(x, s) \text { for some } x \in \Gamma\}
$$

for $0<s<a$. Let $\Delta_{S}$ denote the Laplace-Beltrami operator on the $C^{2}$ surface $S$. The operator $\gamma^{2}-\Delta_{\Gamma}$ is self-adjoint and positive on $L_{2}(\Gamma)$. Set $q=\left(\gamma^{2}-\Delta_{\Gamma}\right)^{-1 / 4}$. Note that $q$ is a tangential pseudo-differential operator of order $-1 / 2$ with $C^{1}$ coefficients.

For $z \in H^{-1 / 2}(\Gamma)$ the expression

$$
\sqrt{\int_{\Gamma}|q z|^{2} d \Gamma}=|q z|_{\Gamma}
$$

is a norm on $H^{-1 / 2}(\Gamma)$ depending on the parameter $\gamma$ which we denote by $|z|_{H_{\gamma}^{-1 / 2}(\Gamma)}$. We have

$$
\frac{1}{\gamma}|z|_{H^{-1 / 2}(\Gamma)}^{2} \tilde{\leq}|z|_{H_{\gamma}^{-1 / 2}(\Gamma)}^{2} \quad \text { for } \gamma \geq 1
$$

where $|\cdot|_{H^{-1 / 2}(\Gamma)}$ denotes a norm in $H^{-1 / 2}(\Gamma)$ which does not depend on $\gamma$.

The operator $q$ can be extended to all of $\Omega$ by defining

$$
q(x, \partial)=\chi(x)\left(\gamma^{2}-\Delta_{\Gamma_{s}}\right)^{-1 / 4}
$$

with $\chi(x)$ a smooth function which is equal to 1 for $x \in \Gamma$ and zero for $x \in \Omega \backslash V$ where $V$ is a tubular neighborhood of $\Gamma$. Note that $q$ is a bounded operator on $L_{2}(Q)$ :

$$
\|q y\|_{Q}^{2} \underset{\leq}{\widetilde{x}}\left\|\frac{1}{\gamma}\right\|_{Q}^{2}, \quad y \in L_{2}(Q) .
$$

Similarly, the normal vector field $\nu$ on $\Gamma$ can be extended as a $C^{1}$ vector field to all of $\Omega$ in such a way that $\nu$ is normal to each surface $\Gamma_{s}$. We split the operator $A^{j} \partial_{j}$ into its normal and tangential part:

$$
A^{j}(t, x) \partial_{j}=A(t, x) \partial_{\nu}+A_{\tau}(t, x, \partial) \quad \text { where } \quad A(t, x)=A^{j}(t, x) \nu_{j}(x) .
$$

We can assume that $A$ is invertible in the collar of the boundary since the boundary is non-characteristic, and that $A^{-1}$ is symmetric.

THIRD STEP: The multiplier $A^{-1} E q v$. We multiply equation (1.1) by $e^{-\gamma t}$ and apply the operator $q$. We obtain

$$
\begin{aligned}
E \partial_{t} w & +\gamma E w+A \partial_{\nu} w+A_{\tau}(\partial) w+[q, E] \partial_{t} v \\
& +\gamma[q, E] v+\left[q, A \partial_{\nu}\right] v+\left[q, A_{\tau}(\partial)\right] v+q B v=q e^{-\gamma t} f
\end{aligned}
$$

where $w=q v$ and $[a, b]=a b-b a$ denotes the commutator of the operators $a$ and $b$. We set

$$
L v=[q, E] \partial_{t} v+\gamma[q, E] v+[q, A] \partial_{\nu} v+\left[q, A_{\tau}(\partial)\right] v,
$$


and using commutator estimates for operators with $C^{1}$ symbols [T91, Proposition 4.1.C], we observe that

$$
\|L v\|_{Q} \underset{\leq}{\widetilde{\gamma}}\|v\|_{Q}
$$

We take the inner product on $L_{2}(Q)$ of equation (2.5) with $A^{-1} E w$ :

$$
\begin{aligned}
\left(E \partial_{t} w, A^{-1} E w\right)_{Q} & +\gamma\left(E w, A^{-1} E w\right)_{Q}+\left(\partial_{\nu} w, E w\right)_{Q} \\
& +\left(A_{\tau}(\partial) w, A^{-1} E w\right)_{Q}+\left(q B v, A^{-1} E w\right)_{Q} \\
& +\left(L v, A^{-1} E w\right)_{Q}=\left(q e^{-\gamma t} f, A^{-1} E w\right)_{Q} .
\end{aligned}
$$

The most interesting term is the third one. Integration by parts gives

$$
\left(\partial_{\nu} w, E w\right)_{Q}=\frac{1}{2}\langle w, E w\rangle_{\Sigma}-\frac{1}{2}\left(w,\left[\partial_{\nu} E\right] w\right)_{Q}
$$

and we note that

$$
\langle w, E w\rangle_{\Sigma} \widetilde{\geq} \int_{\Sigma}|w|^{2}=|v|_{L_{2}\left(0, T ; H_{\gamma}^{-1 / 2}(\Gamma)\right)}^{2}
$$

since $E$ is positive definite. Hence we can use formula (2.6) to estimate the norm $|v|_{L_{2}\left(0, T ; H_{\gamma}^{1 / 2}(\Gamma)\right)}^{2}$. We have

$$
\left|\left(A_{\tau}(\partial) w, A^{-1} E w\right)_{Q}\right| \widetilde{\leq}\|v\|_{Q}^{2}
$$

where we use the fact that $A_{\tau}(\partial)$ is a tangential operator in the collar and

$$
\gamma\left|\left(E w, A^{-1} E w\right)_{Q}\right| \widetilde{\leq}\|v\|_{Q}^{2}
$$

The first term in (2.6) is

$$
\begin{aligned}
\left(E \partial_{t} w, A^{-1} E w\right)_{Q}= & \frac{1}{2} \int_{0}^{T} \partial_{t}\left(E w, A^{-1} E w\right)_{\Omega} d t-\left(\left[\partial_{t} E\right] w, A^{-1} E w\right)_{Q} \\
& -\frac{1}{2}\left(E w,\left[\partial_{t} A^{-1}\right] E w\right)_{Q}
\end{aligned}
$$

and allows the estimate

$$
\left|\left(E \partial_{t} w, A^{-1} E w\right)_{Q}\right| \widetilde{\leq} \frac{1}{\gamma}\left(\|v(T)\|_{\Omega}^{2}+\|v(0)\|_{\Omega}^{2}\right)+\frac{1}{\gamma}\|v\|_{Q}^{2} .
$$

The lower order terms in (2.6) do not cause any trouble:

$$
\left|\left(L v, A^{-1} E w\right)_{Q}\right| \widetilde{\leq}\|v\|_{Q}^{2}, \quad\left|\left(q B v, A^{-1} E w\right)_{Q}\right| \leq \frac{1}{\gamma}\|v\|_{Q}^{2},
$$

and neither does the right-hand side:

$$
\left|\left(q e^{-\gamma t} f, A^{-1} E w\right)_{Q}\right| \widetilde{\leq} \frac{1}{\gamma}\left\|e^{-\gamma t} f\right\|_{Q}\|v\|_{Q} \widetilde{\leq} \frac{1}{\gamma^{2}}\left\|e^{-\gamma t} f\right\|_{Q}^{2}+\|v\|_{Q}^{2} .
$$

Putting everything together we arrive at the estimate

$$
|v|_{L_{2}\left(0, T ; H_{\gamma}^{-1 / 2}(\Gamma)\right)}^{2} \widetilde{\leq}\|v\|_{Q}^{2}+\frac{1}{\gamma^{2}}\left\|e^{-\gamma t} f\right\|_{Q}^{2}+\frac{1}{\gamma}\left(\|v(T)\|_{\Omega}^{2}+\|v(0)\|_{\Omega}^{2}\right),
$$


which proves Theorem 1.1 when combined with the basic estimate (2.2) in view of (2.3) and (2.4).

2.2. Proof of Theorem 1.2. For the characteristic case we can assume that $A$ is in (block-)diagonal form, i.e.

$$
A=\left(\begin{array}{cc}
0 & 0 \\
0 & \widetilde{A}
\end{array}\right)
$$

where $\widetilde{A}$ is a non-singular symmetric $N^{\prime} \times N^{\prime}$ matrix. Instead of the multiplier $A^{-1} E w$ we will now use

$$
\left(\begin{array}{cc}
0 & 0 \\
0 & \widetilde{A}^{-1}
\end{array}\right) E w
$$

The proof is now the same as for the previous theorem except for

$$
\left(\begin{array}{cc}
0 & 0 \\
0 & \widetilde{A}^{-1}
\end{array}\right)\left(\begin{array}{cc}
0 & 0 \\
0 & \widetilde{A}
\end{array}\right)=\Pi
$$

where $\Pi$ is the projection onto $(\operatorname{Ker} A)^{\perp}$.

REMARK. The proof shows that the regularity assumptions on the coefficients can be weakened. $C^{1}$ regularity of $E$ and $A^{j}$ is necessary only near the boundary $\Sigma$, whereas in the interior, $E, \partial_{t} E, A^{j}, \partial_{j} A^{j} \in L_{\infty}$ will suffice.

Acknowledgements. The author is grateful for the comments of an anonymous referee who pointed out a number of errors in the original manuscript.

\section{References}

[CP82] J. Chazarain and A. Piriou, Introduction to the Theory of Linear Partial Differential Equations, North-Holland, Amsterdam, 1982.

[C96] M. Cessenat, Mathematical Methods in Electromagnetism, World Sci., Singapore, 1996.

[EM02] M. M. Eller and J. E. Masters, Exact boundary controllability of electromagnetic fields in a general region, Appl. Math. Optim. 45 (2002), 99-123.

[F54] K. O. Friedrichs, Symmetric hyperbolic linear differential equations, Comm. Pure Appl. Math. 7 (1954), 345-392.

[G01] A. Glasman, On the regularity of solutions of the Maxwell dynamical system, PDMI-preprint, 2001.

[K70] H.-O. Kreiss, Initial boundary value problems for hyperbolic systems, Comm. Pure Appl. Math. 23 (1970), 277-298.

[LT90] I. Lasiecka and R. Triggiani, Sharp regularity theory for second order hyperbolic equations of Neumann type. I: $L_{2}$ nonhomogeneous data, Ann. Mat. Pura Appl. (4) 157 (1990), 285-367. 
[LT91] I. Lasiecka and R. Triggiani, Regularity theory of hyperbolic equations with non-homogeneous boundary conditions II. General boundary data, J. Differential Equations 94 (1991), 112-164.

[LT94] -, 一, Recent advances in regularity of second-order hyperbolic mixed problems and applications, in: Dynamics Reported, Exp. Dynam. Systems 3, C.K.R.T. Jones et al. (eds.), Springer, Berlin, 1994, 104-162.

[LP60] P. D. Lax and R. S. Phillips, Local boundary conditions for dissipative symmetric linear differential operators, Comm. Pure Appl. Math. 13 (1960), 427-455.

[MO75] A. Majda and S. Osher, Initial-boundary value problems for hyperbolic equations with uniformly characteristic boundary, ibid. 28 (1975), 607-675.

[M73] S. Miyatake, Mixed problem for hyperbolic equation of second order, J. Math. Kyoto Univ. 13 (1973), 435-487.

[M00] G. Métivier, The block structure condition for symmetric hyperbolic systems, Bull. London Math. Soc. 32 (2000), 689-702.

[MZ05] G. Métivier and K. Zumbrun, Hyperbolic boundary value problems for symmetric systems with variable multiplicities, J. Differential Equations 211 (2005), 61-134.

[S70] R. Sakamoto, Mixed problems for hyperbolic equations. I. Energy inequalities, J. Math. Kyoto Univ. 10 (1970), 349-373.

[T98] D. Tataru, On the regularity of boundary traces for the wave equation, Ann. Scuola Norm. Sup. Pisa Cl. Sci. (4) (1998), 185-206.

[T91] M. Taylor, Pseudodifferential Operators and Nonlinear PDE, Birkhäuser, Boston, 1991.

Department of Mathematics

Georgetown University

Washington, DC 20057, U.S.A.

E-mail: mme4@georgetown.edu

Received on 10.12.200\%;

revised version on 26.3.2008 
